\title{
Enhancement of professionalism during postgraduate training in Pediatrics
}

\author{
Shaw, S.C. ${ }^{1}$, Salagre, S.B. ${ }^{2}$, Mukherjee, S. ${ }^{1}$, Adhikari, K.M. ${ }^{1}$
}

\section{Introduction}

Professionalism is an essential element of medicine's contract with society. The medical professionals are expected to display professional attitudes, behavior, values and ethics in their day to day practice. It is important that we inculcate these values in our students from the very beginning, as they are actively involved in dealing with patients. Though literature exists on the subject, the concept of professionalism in medical education and its implementation is evolving and there is a felt need to put in place robust method of education or assessment in relation to professionalism.

\section{Objectives}

The objective of the study was to assess enhancement of professionalism in postgraduates in pediatrics through Professional Mini Evaluation Exercise (P-MEX), and Jefferson physician empathy score by introducing a teaching-learning module for professionalism encompassing interactive workshop, role modelling, and feedback to the students.

\section{Methodology}

The study was Interventional and the subjects were the MD residents in Pediatrics in first year,

${ }^{1}$ Department of Pediatrics, Armed Forces Medical College, Pune, India

${ }^{2}$ Department of Medicine, Seth GS Medical College and K.E.M. Hospital, Mumbai, India

Corresponding author: Dr Subhash Chandra Shaw drscshaw@rediffmail.com

DOI: http://doi.org/10.4038/seajme.v15i2.347 second year, and third year, 6 in each year (total 18 in number). After obtaining institutional ethical clearance, we planned the professionalism module including Interactive workshop on

professionalism (conducted over half a day, including lectures, role plays, videos, case studies), role modelling by faculty during clinical rounds/OPDs and reflective writing sessions by $P G$ students after witnessing any critical event. The plan of interactive workshop on key elements of professionalism (altruism, accountability, duty, excellence, honor, integrity and respect for others) was demonstrated and discussed with faculty of medical education as well as faculty of department of Pediatrics of the institute and was validated. All the faculty of department of Pediatrics were sensitized and encouraged to demonstrate role modelling in their daily rounds. They were urged to be aware of being a role model, demonstrate clinical competence, protect time for teaching, show a positive attitude, implement a student centered approach to teaching, facilitate reflection on clinical experiences and what has been modelled, encourage dialogue with colleagues and work to improve the institutional culture and finally whenever possible to be explicit about what is being modelled. Baseline P-MEX score, and Jefferson's empathy scale was scored for all the residents which was repeated again after 3 months of the workshop, as the role modelling continued and feedback to the participants continued. Reflections were also sought after witnessing any critical event during the study period. At the end of the study, feedback from faculty and residents were taken about the entire process. Finally collected data were analyzed and pre and post data were compared by Wilcoxon Signed-Ranks Test. 


\section{Results}

As assessed by Professionalism Mini-Evaluation Exercise (PMEX), the median post-test scores were statistically significantly higher than the median pre-test scores except in admitting errors/omissions and in avoiding derogatory language where there was no difference noticed. For rest all the parameters, the students could meet or exceeded expectations. However, Jefferson's Empathy score for physicians revealed that the median post test scores were similar and not statistically different from the median pre-test scores. Feedback received from the participants was that they liked the module and they felt that these concepts should be introduced at even undergraduate level, and the workshop on professionalism be repeated at yearly intervals.

\section{Discussion}

Teaching professionalism is not akin to imparting a technical clinical skill. Professionalism is learned most effectively through the influence on students of clinicians they encounter in the course of their education (role models), thus possibly, role modelling and mentoring are the most effective techniques for developing professionalism (Goldie J, 2013; Birden $\mathrm{H}$ et al, 2013). Similarly, there is hardly any reasonable gold standard for its assessment $(\mathrm{Li} \mathrm{H}$ et al, 2017). In our study, there was a significant change noticed in PMEX, but not in Jefferson's Empathy score. The reason could be that empathy is often intuitive thus less amenable to change unlike other aspects of professionalism, and embodying empathy is a time and exposure related experiential learning which is often individual dependent. Strengths of the study was the novelty, and relevance of the study. The limitation of the study was that the role modelling by the teachers was difficult to measure and ensure.

\section{Conclusion}

Concept of professionalism among postgraduates can be enhanced through sensitization interactive workshop, and role modelling.

\section{References}

Birden, H., Glass, N., Wilson, I., Harrison, M., Usherwood, T., Nass, D. (2013) Teaching professionalism in medical education: a Best Evidence Medical Education (BEME) systematic review. BEME Guide No. 25. Med Teach, 35, 7, e1252-66.

Goldie, J (2013) Assessment of professionalism: a consolidation of current thinking. Med Teach, 35, 2, e952-6.

Li, H., Ding, N., Zhang, Y., Liu, Y., Wen, D. (2017) Assessing medical professionalism: A systematic review of instruments and their measurement properties. PLOS ONE, 12, 5, e0177321. 